\title{
Interruption and Pausing of Public Display Games
}

\author{
Tiare Feuchtner ${ }^{1}$ \\ Robert Walter ${ }^{2}$ \\ Jörg Müller ${ }^{1}$ \\ ${ }^{1}$ Department of Computer Science, Aarhus University \\ ${ }^{2}$ Quality and Usability Lab, Telekom Innovation Laboratories, TU Berlin
}

\begin{abstract}
We present a quantitative and qualitative analysis of interruptions of interaction with a public display game, and explore the use of a manual pause mode in this scenario. In previous public display installations we observed users frequently interrupting their interaction. To explore ways of supporting such behavior, we implemented a gesture controlled multiuser game with four pausing techniques. We evaluated them in a field study analyzing 704 users and found that our pausing techniques were eagerly explored, but rarely used with the intention to pause the game. Our study shows that interactions with public displays are considerably intermissive, and that users mostly interrupt interaction to socialize and mainly approach public displays in groups. We conclude that, as a typical characteristic of public display interaction, interruptions deserve consideration. However, manual pause modes are not well suited for games on public displays. Instead, interruptions should be implicitly supported by the application design.
\end{abstract}

\section{Author Keywords}

Public displays; games; pause mode; interruption; gesture interaction; user studies;

\section{ACM Classification Keywords}

H.5.m. Information Interfaces and Presentation (e.g., HCI): Miscellaneous

\section{INTRODUCTION}

Public displays are being used for a variety of purposes, including civic participation [7, 31], information dissemination $[23,29]$, social cohesion and community building [25, 30], advertising and community support [1], and social games [17]. Interaction with such displays poses a number of unique challenges, among them display blindness [19], interaction blindness [18, 23], immediate usability, and gesture teaching $[33,34]$. In this paper we want to raise attention to another such challenge: users frequently interrupt interaction, be it out of their own accord, or induced by the environment. We believe that this has implications for the design of public display applications that require continuous input, such as games.

Permission to make digital or hard copies of all or part of this work for personal or classroom use is granted without fee provided that copies are not made or distributed for profit or commercial advantage and that copies bear this notice and the full citation on the first page. Copyrights for components of this work owned by others than the author(s) must be honored. Abstracting with credit is permitted. To copy otherwise, or republish, to post on servers or to redistribute to lists, requires prior specific permission and/or a fee. Request permissions from Permissions@ acm.org.

MobileHCI '16, September 06 - 09, 2016, Florence, Italy

Copyright is held by the owner/author(s). Publication rights licensed to ACM.

ACM 978-1-4503-4408-1/16/09\$15.00 DOI: http://dx.doi.org/10.1145/2935334.2935335

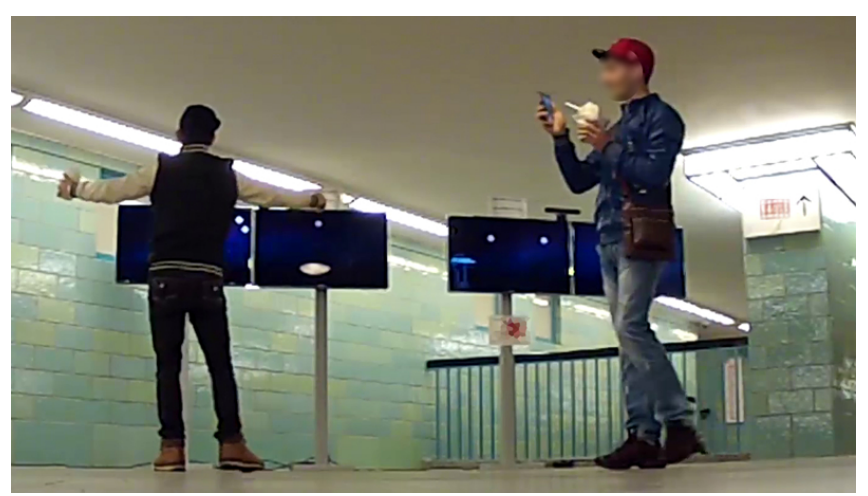

Figure 1. In this field study we observed that users sometimes interrupt their interaction with a public display game. Here a user can be seen filming his friend with his phone and holding something to eat in his other hand, while in the background his game continues.

It seems fundamental to have a safe way to temporarily disengage from, i.e., interrupt, a continuous interaction. Therefore pause modes exist in most types of video games, where they are most frequently triggered by pressing a key or button. For gesture controlled games certain gestures can be defined to trigger pauses, as for instance the Pause gesture supported by the Kinect ${ }^{1}$. These pausing techniques have prevailed for games that are played in a non-public environment, e.g., a living room, or even on personal, portable devices in public. However, they all make the assumption that the game is the user's main activity at the time, receives regular attention, and that the user has the opportunity to learn the controls. Games on public displays often do not fulfill these assumptions: interactions are mostly spontaneous, first-time interactions are frequent, and a multitude of distracting factors in the public setting and social context compete for the user's attention. We set out to explore if this intermissive nature of interaction could be supported by adequate pausing techniques.

To understand interruptions and design techniques for pausing continuous interaction with a public display we first performed a video analysis of existing public display installations, finding that users indeed interrupt their game-play on a public display (avg. 1.48 interruptions per user session). Motivated by this finding, we iteratively developed a simple Kinect game with four pausing techniques: Modal Pausing, Quasimodal Pausing, Quasimodal Idling, and Amodal Activation. To compare these techniques and investigate pausing behavior in detail, we then conducted a 10-day field study, where we installed our Kinect game on a set of public displays in the passage hall of a highly frequented subway station (see Figure 1). In our field study, we explored how fre-

\footnotetext{
${ }^{1} \mathrm{http}: / / \mathrm{www} . \mathrm{microsoft.com/en-us/kinectforwindows}$
} 
quently users interrupt interaction, analyzed reasons of interruptions, and evaluated the effectiveness of the above pausing techniques in supporting these interruptions.

The mechanics of our game resemble those of the well known game Space Invaders ${ }^{2}$, where users control their spaceship with their body. The game affords multiple users simultaneously and can easily be altered from a continuous game-flow to a pauseable game, without affecting significant aspects of the game, such as its goal or mechanics. By thus adapting the degree of attention and continuity of input required from the users, we allowed them to freely manage the attention dedicated to game-play versus social interaction within their group.

With this work we contribute to both the understanding of user behavior and the design of public display games. We provide results from a quantitative and qualitative evaluation of interruptions in public display interaction with a multiuser game. Furthermore, we propose a design space of pause modes and developed four pausing techniques to explore their application in an extensive field study. We believe that designers of continuous public display applications, such as games, need to pay special attention to handling interruptions of interaction by the user, and that the details of this behavior warrant further research in this understudied field.

\section{RELATED WORK}

The related work discussed in this section covers research on public display installations, games in terms of casual gameplay and pausing, and the definition of social groups and group behavior.

\section{Public display installations}

Public displays have been a topic of research in humancomputer interaction for a long time. An excellent overview of work until 2003 is given in [21]. More recently, thorough research on the difficulty of conveying interactivity and enticing interaction shows that on-screen content and positioning of the display play an important role [12], and that displaying a mirror image or silhouette of the approaching user is more successful in enticing interaction than using avatars [18]. Furthermore, users more readily acknowledge a public display,

\footnotetext{
${ }^{2}$ http://en.wikipedia.org/wiki/Space_Invaders
}

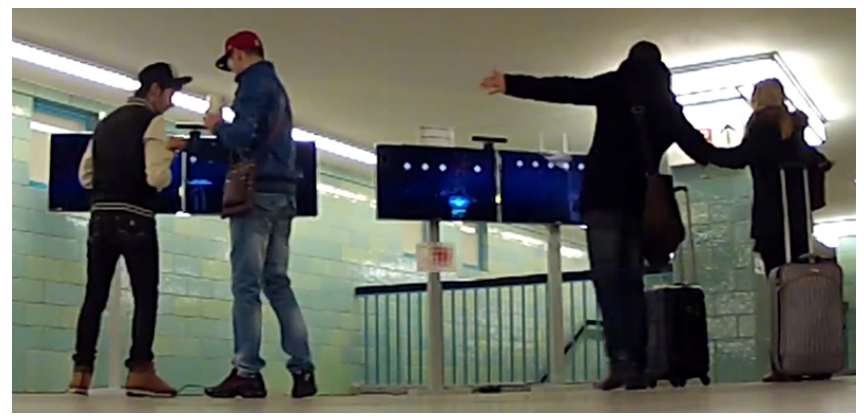

Figure 2. On the left a user tugs at his friend's sleeve interrupting both of their interactions. He also carries food in his hand, of which he occasionally takes a bite. and in particular its interactivity, when another user is already engaged with it $[5,17]$ (know as the honeypot effect). On the subject of shared public displays it was found that most frequently users approached the screen in pairs and then either worked individually, each occupying part of the screen space, or in cooperation [25]. In the context of sustaining interaction, ease of use, playfulness, and novelty are mentioned as the main incentive for users to interact [13].

\section{Games on public displays}

Playful interactions and games are intrinsically motivating and therefore present popular content for public displays. Most games on public displays do not involve continuous interaction: they are not fast paced, don't require undivided attention, and allow spontaneous joining or leaving of players (e.g., Flashlight Jigsaw [6], The Red Nose Game [22]). Others have very short play sessions, requiring only bursts of intense interaction (Drunken Ed [3]). If longer interactions with more engaging, attention-gripping content are to be achieved, the requirement of a pause mode becomes evident. Furthermore, public displays frequently support multiple users, which makes interruptions very likely: while researching interactional trajectories, Flintham et al. [9] report on "frequent dis- and re-engagement" of users with Flypad, a game installed as part of a permanent exhibition in an art gallery. The authors describe how users repeatedly engaged and disengaged with the installation, specially within groups. Players were taking turns on each others' pads, or even temporarily sustained someone's interaction if they shortly vacated their station. A few other publications on public displays mention such interruptions briefly $[6,18,25]$. However, we are not aware of any prior work properly addressing the question of pausing and interrupting behavior.

\section{Games}

Although the problem of pausing interaction does not only apply to games, we focus on playful interactions. We decided to use a game as apparatus to investigate our research questions and therefore wish to briefly review the topic of casual games and pausing.

\section{Casual games}

Kultima [15] mentions Casual Games as a category that may include games of all genres. Some typical characteristics for casual games are short play sessions, cheerful experiences, safe themes, familiar controls, low levels of immersion, and low cost. Examples may be browser games, or games on mobile devices. One major aspect of casual games is Flexibility, which means permitting the game to be a secondary activity by allowing interruptions and forgiving errors. For this reason we argue that games on public displays necessarily need to be casual games, as is further supported by [6].

\section{Pauses in games}

The pause mode is a basic function in most video games that we generally take for granted. It allows the player to temporarily exit the game and resume it after an indefinite amount of time without endangering her performance. Since we were not able to find any research on this topic, we performed a short survey of popular video games to get a more structured view of how and when pausing is realized. 
From looking at a range of action games that require continuous input (e.g., platformers, shooters, RPGs), we discovered that nearly all single player and local multiplayer games have a pause mode (e.g., Super Mario, Zelda, Little Big Planet, MarioKart, Assassins Creed, GTA, Limbo). Some of the multiplayer games impose that only a certain player may pause and resume (e.g., Player 1, or the host), or that only the person who paused the game has the power to resume. On the other hand, arcade games or online multiplayer games can usually not be paused and some even penalize people for "leaving" the game in mid-match (e.g., League of Legends). In other online multiplayer games (e.g., Diablo) a player can "pause" to access her inventory, but her avatar will remain in the game and be vulnerable to the actions of other players or enemy AIs. And while games on mobile devices sometimes don't feature an explicit pause button, most enter a pause mode implicitly when interrupted by a user action (e.g., when accessing the game menu, switching to the home screen or another app).

The pause mode is an interruption the system knows of and can be considered in the game. The same applies to some other types of interruptions, like loading, lost connection, low battery, advertisement, a call on the phone, etc. Interruptions the system doesn't know of are more difficult to handle, such as when the player dedicates her attention to social interaction, or other events in the environment (e.g., getting up to answer the door, getting on or off a bus). The reasons for pausing or interrupting a game vary strongly, depending on the game type, the device, and the environment the game is played in. PC or console games are typically more complex and we assume that users most often pause to change settings, save the current progress, or consider options. Portable gaming devices (e.g., GameBoy, Nintendo 3DS, Smartphone, Tablet) on the other hand often serve as a means of entertainment when we are in a public environment, which is a source of frequent disturbances (e.g., social encounters). Mobile games are additionally prone to interruptions due to the phone's main function of being a communication device (i.e., receiving messages and calls).

\section{Social groups}

When engaging in interaction with public displays, people usually approach the installations in groups and remain in social interaction with each other [13, 20, 25]. For this reason we wish to briefly address the subject of social groups.

In sociology a group is defined as two or more people who interact with each other and are interdependent in regard to their individual goals and needs $[2,28]$. In a wider sense, they have a common goal, which can be as simple as walking to the same bus station. Further expanding on groups, Goffman defines a "social gathering" as a "full set of persons mutually present to one another during any one continuous period of time" [10]. This term applies to people who are simultaneously interacting with, or observing, a public display. The individuals in this social gathering inherently agree on new rules of behavior (e.g., gesturing, dancing) and courtesy (e.g., taking turns, cooperating, or competing) and thus become a small "social system" with specific roles. In conjunction with public displays, several authors categorize actors and spectators $[6,8,11,22]$ into roles such as teacher and student, or clown and audience [20,25]. They hereby refer to users taking the leading role of instructing their companions in the use of the installation, or taking the stage to entertain them with performative behavior. In this paper we borrow some of the terminology and refer to users when speaking of both actors and spectators together, and explicitly use actors only when discussing users who at some point interact with the public display.

\section{Summary}

Although the fact that users of public displays interrupt interaction has been established in several publications, this topic has not yet been investigated in detail. In particular, we are not aware that pausing and interruption behavior has been analyzed in a quantified manner and previous work can only let us guess at the answers to our research questions, i.e., if users interrupt interaction (RQ1), what for (RQ2), and how pause gestures could be realized (RQ3). We believe that this is a highly relevant topic for public display research and application design, because displays not adequately handling interruptions might not leverage their full potential. We aim at making a contribution to fill this gap in the literature by providing the first qualitative and quantitative investigation of interruptions in interaction with public display games, proposing potential techniques to support this behavior, and evaluating them in a field study.

\section{META-ANALYSIS OF INTERRUPTIONS}

As a first step to gain insights about interruptions during interaction with continuous applications on public displays, we analyzed data from our previous installations Looking Glass [18] and StrikeAPose [33], both of which offered playful interaction with basic physics simulations. Looking Glass was installed in a shop window and invited passers-by, who were tracked by a Kinect, to interact from the sidewalk. The screen showed the user's colored silhouette and several soccer balls, which bounced off of the user's body representation and could thus be played with. StrikeAPose consisted of a large-screen display, which featured a similar body representation and prompted users to assume a certain body pose. If a user complied, he was rewarded with a funny bunny mask, or a hat, displayed on top of his silhouette. This second installation was situated in the entrance hall of a cafeteria on the university campus. We reevaluated a random subset of recordings from the depth cameras analyzing 84 users, with focus on interruptions of interaction and inter-group relations. These users were selected randomly until we could not deduct any further learnings from the data.

\section{Findings \\ Interruptions and turn-taking}

While the overall session time averaged to one minute, the interruptions and times of action most frequently occured in short bursts. We hereby define a session as the time span from when the first individuals of the group halt in front of the screen and direct their attention toward it, until when all members of the group leave the interaction space. Users spent 
more than $80 \%$ of the time playing individually instead of simultaneously, meaning that while one person played the others interrupted their interaction to watch. This type of behavior caused each player to interrupt interaction an average of 1.48 times per session, which adds up to an average of almost 4 interruptions per group (see interaction samples of StrikeAPose in Figure 3). Single users (group of size 1) seemed to interrupt interaction less often per session, perhaps since less turn-taking and social interaction could take place. Such turntaking might have partly been due to the nature of the application and the limited interaction space, but it has also been observed on a very different installation [25]. Our main take from this finding is that users were often not prepared to interact continuously and in those cases a pause mode could be useful.

\section{Group size}

Users generally approached in groups of 2 to 3 people, rarely of 1 or 4 people, and almost never more than 4 . This may have been influenced by the size of both the screen and the interaction space. However, it is also a known phenomenon in social psychology $[2,16]$ and has previously been noted by several authors of related work on public displays [13, 20, 25]. We identify a group as multiple users who are engaged with the public display at the same time (either as actors, or as spectators). Most often the members of a group arrived together and left together, signaling that the group was actually formed previous to the interaction with the display. The common occurrence of groups poses special requirements if a pause mode is to be implemented, since it must be carefully considered how the game is paused and which of the users are affected by this pause.

Overall, the above mentioned findings support our claim that any engagement with public displays is prone to interruptions, which deserve consideration. Furthermore, since users mostly arrive in groups, games on public displays should permit multiple users to interact simultaneously and allow them

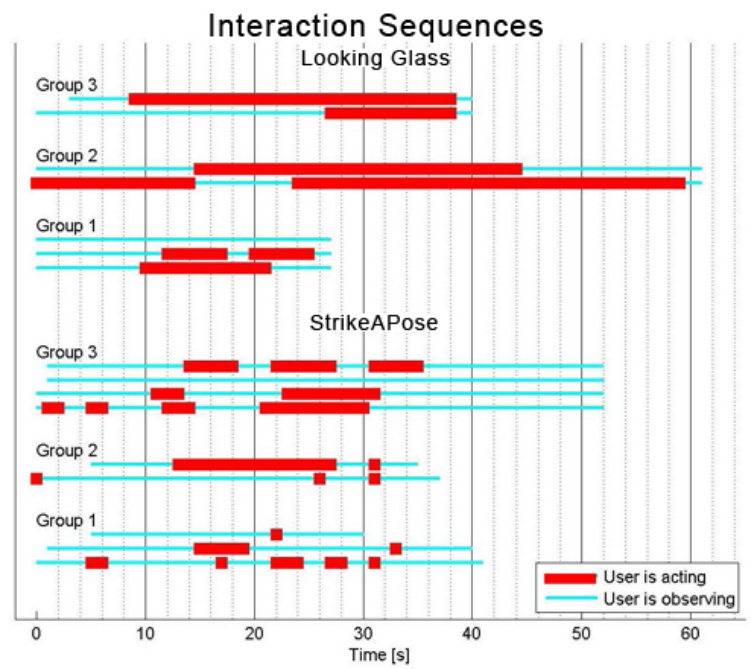

Figure 3. Each person is represented by a line, with the fine stroke indicating spectatorship and the thick stroke indicating interaction. E.g., group 3 of StrikeAPose shows interaction in bursts and turn-taking.

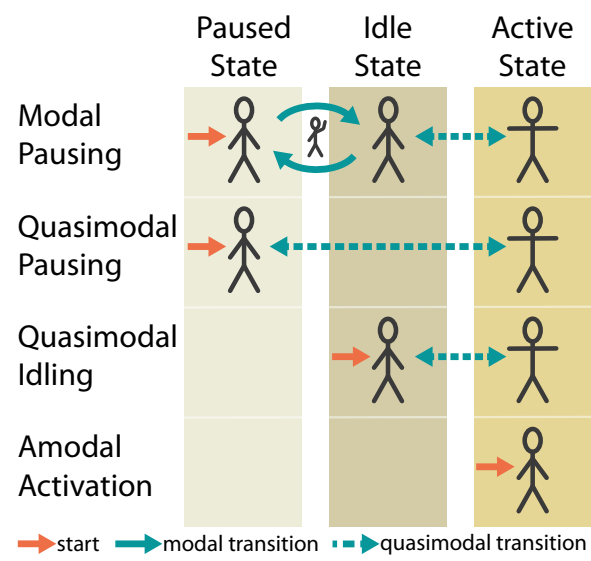

Figure 4. A user can be in one of three states: Paused, Idle or Active. Transitions between these states can be modal (play/pause gesture), or quasi-modal (ready pose).

to smoothly transition from spectator to actor (i.e., Paused to Active) and vice versa.

\section{ITERATIVE DESIGN OF PAUSING TECHNIQUES}

\section{Design space}

In accordance with our findings from our meta-analysis of interruptions, we made a categorization of user states and transition modes that we present below, followed by a set of four techniques we propose to support such transitions. We do not claim that this categorization is complete. It merely contains the dimensions we focus on to explore the efficacy of modal and quasimodal pausing in a game.

\section{Pause vs. interruption}

We add for clarity that we use the term pause to describe the user's game state, when the game is safely interrupted through a voluntary and actively signaled action (e.g., pressing a pause button). In other words, it is an interruption that the system knows of and can respond to. In contrast, we use the term interruption of interaction when we mean that the user ceases to interact with the screen (e.g., she may be observing others, or may also direct her attention elsewhere, be it voluntarily, or caused by external circumstances), without letting the system know about it (i.e., without pausing).

\section{States of interaction}

We define three possible states for a user: (1) Paused, (2) Idle and (3) Active. Paused is when a user has signalled her wish to pause the system and is consequently not able to interact until she unpauses (e.g., when a video game is set to pause). In this state, the user does not affect the application, and the application does not affect the user. Idle is when the user is unpaused, but not actively interacting (the interaction may have been interrupted, or the user may just be observing). The user does not affect the application, but the application may affect the user (e.g., the user's avatar in the game may receive damage). Finally, Active is when the user is unpaused and interacts actively (e.g., by shooting in the game). Here the user and application mutually affect each other. 


\section{Modal and quasimodal transitions}

To describe our pausing techniques we use the terms modal and quasimodal based on the definitions by Jef Raskin [26]. He explains the difference between modal and quasimodal as the distinction between using the Caps Lock key or the Shift key to write capital letters. Pressing (and releasing) the Caps Lock key causes a modal transition of the system from lower case to upper case, and only pressing it again will reverse the state. Using the Shift key, on the other hand, is considered a quasimodal transition, since the system only remains in upper case while the key is being pressed.

\section{Pausing techniques}

We propose four different techniques for exploring modal and quasimodal transition modes, as explained above. Figure 4 provides an overview of the states and transitions of each technique.

1. Modal Pausing: Users start in Paused state and can perform a modal transition from Paused to Idle state and back. We implemented this as a play/pause gesture, or by defining a certain interaction area, which users can enter to transition to Idle. Users can further switch from Idle to Active state through a quasimode, e.g., by performing a ready pose.

2. Quasimodal Pausing: Users start in Paused state and can transition directly to Active state through a quasimode, e.g., a ready pose.

3. Quasimodal Idling: Users start in Idle state and may transition to Active state through a quasimode (e.g., a ready pose).

4. Amodal Activation: Users start in Active state and no transitions are possible. This technique does not offer any opportunity to signalize (un)willingness to play.

Note that techniques 1-3 all require the ready pose and in techniques 3 and 4 no actual pause can be triggered in the game.

\section{Iterative development process}

With the objective of testing our game in the wild and to iteratively design the pausing techniques explained above, we deployed our system on a university campus. We also wished to confirm that the displayed hints were understood by the users, and that the gestures were acceptable, and reliably tracked by the system. Our setup, which can be seen in Figure 5, was installed in the lobby of a building at the UDK (Universität der Künste Berlin). The interaction area was directly visible from the main entrance and allowed interaction without obstructing the way for others. Half of the screen space showed the interactive game, whereas a score board was projected on the other half to inform users about their performance and entice competition.

\section{System}

The system consisted of a back projection screen $(5 \mathrm{~m}$ wide and $2 \mathrm{~m}$ high) with a mounted Kinect camera to detect passersby in the interaction area. The application was implemented in Java, using OpenNI ${ }^{3}$ and NITE libraries to access the

\footnotetext{
${ }^{3}$ http://structure.io/openni
}

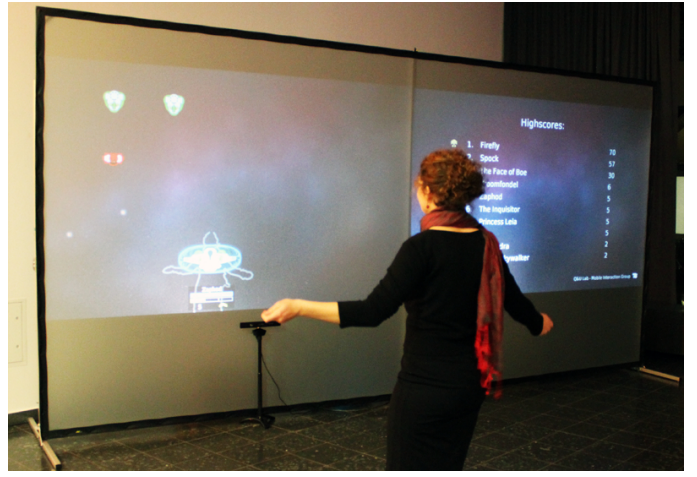

Figure 5. Deployment in the lobby of a university building with a back projection screen showing the game (left) and a highscore list (right).

Kinect data. The depth image video and screen capture were recorded and later analyzed with a custom annotation tool that was built for this study using Python and the VLC library ${ }^{4}$.

\section{Game design}

We intended to design a game, which fulfills the requirements of supporting multiple simultaneous users and affording to be manually paused, without inherently changing the nature and goal of the game. We adopted the concept of Space Invaders, where attacking ships must be shot down, while avoiding being hit by their missiles in turn. Tracked users are represented by their silhouettes [18] with a spaceship attached to their shoulders. Enemies appear in waves and repeatedly swipe across the screen in horizontal direction. Shots are fired from the wings of the users' spaceships at predefined intervals, with which they must destroy the enemy ships to increase their score. The users can move the spaceship along the horizontal axis by walking left and right in order to hit targets and dodge bullets. A number of power-ups are randomly dropped by defeated enemies to motivate exploration and longer interaction. The users can catch these power-ups with their spaceships to change or upgrade their weapon, or to recover their protective shield.

\section{Techniques}

Of the possible implementations of the techniques mentioned in the previous section, we included the following four in our deployment: Modal Pausing with play/pause gesture, Modal Pausing with active area, Quasimodal Pausing and Quasimodal Idling with a ready pose. The active area defines a specific space in front of the screen that allows a transition from "implicit to explicit interaction" [32], according to the notion that spectators often keep a greater distance to the display, while actors move closer [31].

\section{Implementation of pauses}

To allow ongoing interaction in this multiuser game, pauses are triggered for each user individually. Tracked users are represented by their silhouettes, and users who are Active or Idle (unpaused) additionally have a spaceship that is vulnerable to enemy fire. When pausing the game the spaceship vanishes, but users do not lose their player data (e.g., assigned

\footnotetext{
${ }^{4}$ https://wiki.videolan.org/Python_bindings
} 


\section{A RAISE YOUR HAnD to PLAY! \\ B RAISE YOUR HanD

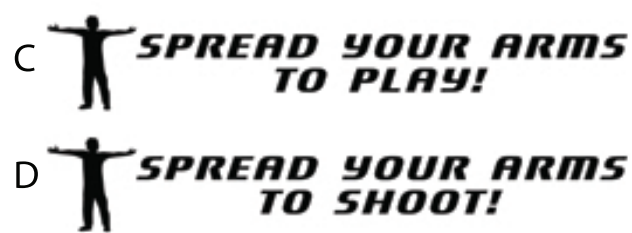

Figure 6. Hints informing about how to toggle pause in Modal Pausing technique (A,B), how to play in Quasimodal Pausing technique (C), and how to perform the shooting pose in both Quasimodal Idling and Modal Pausing technique (D).

name and score), as opposed to when leaving the tracking area.

\section{Choice of hints and gestures}

While the pause button is omnipresent on hand-held gaming devices and game controllers, in PC games pause is often triggered with a specific key on the keyboard, and on touch screen devices it might involve tapping a button. Somewhat more unusual are voice commands, as are supported by the XBoxOne. Gesture based interaction however brings along the challenge of designing a new pause command without the use of buttons or speech. And unfortunately there does not seem to be an intuitive, universal gesture for pausing. The Kinect ${ }^{5}$ has established the Guide Gesture (extending an arm at $45^{\circ}$ from the body) to pause video games, which seems rather awkward. One of the rare accounts about pause gestures in a scientific publication shortly mentions the use of a T-pose for pausing an exergame [4]. This publication goes on saying that the gesture was found confusing for this purpose and that pause was never triggered intentionally.

In contrast to using the T-pose for pausing, we use it to actively play, since spreading the arms to fly the spaceship also nicely fits our game design. And for toggling a pause we chose the raise-hand gesture. Both gestures permit sufficiently reliable tracking, support performative learning [25, 27], and are distinct enough from typical body language to prevent inadvertent triggering.

In order to inform users about the required actions, flashing hints were displayed above their silhouettes. Two gestures/poses were to be learned in this manner:

- play/pause gesture: for the modal transition we used a raise-hand gesture (see Figure 6, A and B).

- ready pose: for the quasi-modal transition we used the $T$ pose (spreading both arms as if flying; see Figure 6, C and D).

In accordance to the findings of Kukka [14] and Walter [33], the hints consisted of animated icons with text alternatingly shown in English and German.

\section{Findings}

During the 9-day deployment in the lobby of the university building 1516 people were tracked by the Kinect camera and live observations were made throughout most of the days. We found that the game was well received, quickly understood, and the hints to perform the T-pose and raise-hand gesture

\footnotetext{
${ }^{5}$ http://support.xbox.com/en-US/xbox-360/kinect/body-controller
}

were readily followed. We also found that the implementation of the Modal Pausing technique with an interaction area (which allowed users to transition between Paused and Idle state by moving to different regions of the tracking area), was not used for this purpose by any users. And since the range of the Kinect is limited, subdividing the interaction area left only little room for active users. These findings led us to remove this implementation from our field study, which instead included the Amodal Activation technique.

\section{FIELD STUDY}

The objectives of our field study were to quantify the number and duration of interruptions, to investigate their reasons, and to explore the consequences of our pause techniques. Furthermore, we were interested in how groups influenced interaction and interruptions. Aiming for higher ecological validity compared to a university campus, we performed our main field study in a major train and subway station.

\section{Deployment}

We deployed two pairs of 40" screens on stands, each with a mounted Kinect and a laptop strapped on in the back. The setup was positioned in a strongly frequented entry and passage hall of the subway station Alexanderplatz. This is one of the largest subway stations in Berlin, where 10 subway and train lines cross with a dozen bus and tram lines, and many people pass through daily on their way to work or school. As a major landmark of Berlin it is also strongly frequented by tourists and, since the study took place in December, the Christmas market at Alexanderplatz was a magnet for visitors. The public display was set up a in corner where it was directly passed by passengers arriving with the subway line 5 (Alexanderplatz is the terminal station on this line), and clearly visible to people transferring to line 8 , or passing on towards the exit. The main walking direction of passers-by led from left to right directly through the interaction area (see setup in Figure 7).

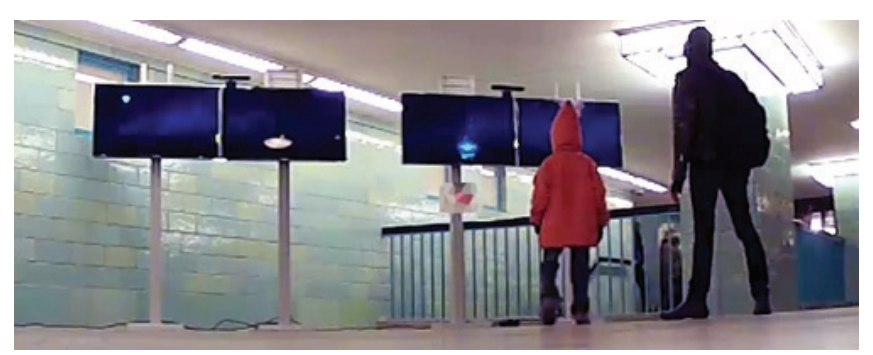

Figure 7. Two screen pairs were set up in the subway station, with a Kinect mounted on top of each to track users. 


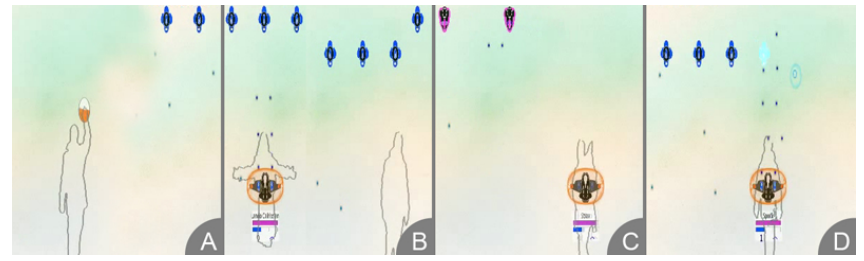

Figure 8. (A) Modal Pausing technique: User in Paused performing raise-hand gesture to unpause; (B) Quasimodal Pausing technique: User doing T-pose (Active state) next to user in Paused; (C) Quasimodal Idling technique: Idle user; (D) Amodal Activation technique: User in Active state.

\section{Experimental design}

The main focus of our field study was to answer the following three research questions:

RQ1 Do users sometimes interrupt their interaction with the public display?

RQ2 Why do users interrupt their game-play?

RQ3 Are the above mentioned pausing techniques effective in supporting users' interruptions?

Our independent variable was the technique, which switched every 17 minutes to one of the following values: Modal Pausing technique (with raise-hand gesture), Quasimodal Pausing technique (with T-pose), Quasimodal Idling technique (with T-pose), and Amodal Activation (amodal). The switching time was chosen to avoid repetition of the same techniques at the time of train arrivals. The implementation of states across the different techniques can be seen in Figure 8, where paused users are shown only as silhouettes, while Idle users receive a spaceship, and users who are Active shoot. Our quantitative dependent variables were the number of interruptions, the interruption duration, the interaction duration, and the duration of stay. We also evaluated the interactions qualitatively through observations and interviews.

\section{Data analysis}

User actions registered by OpenNI were logged and this data was used to discern passers-by from people who stopped to interact with the screen. Further, the Kinect's depth video and screen capture were recorded for analysis through manual video annotation. To gain a full view of the interaction area, the annotation tool shows a grid of 4 videos with synchronized screen captures and depth images of both screen sets (Figure 9). The tool loads a user list and allows to navigate to specific users by jumping to their arrival time-stamp. The right sidebar is reserved for annotation buttons and text fields for notes. Apart from marking the times when users entered and left the interaction space, we also noted the start and end times of observation, interaction, interruptions, and actions, such as raising a hand, or spreading the arms. Further information could be added, such as the observed group size, user behavior during interruptions, or if the user was carrying items. We also made notes of situations when something interesting or unusual happened, such as users dancing, helping, or tackling each other. Users who were affected by a change of technique during their interaction session were

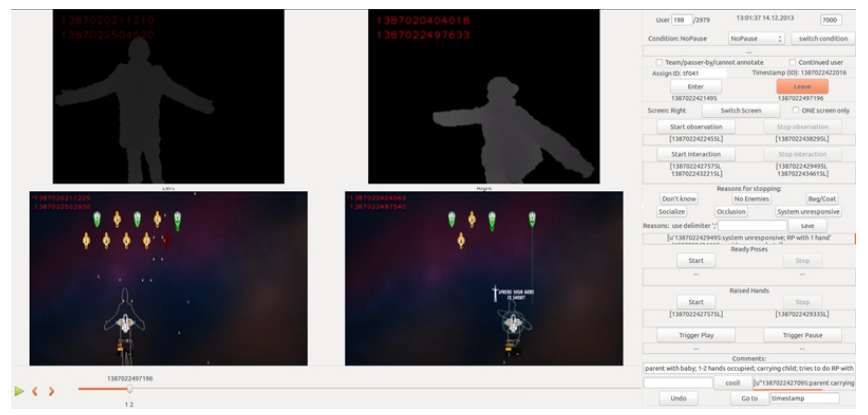

Figure 9. Annotation tool with 4 synchronized videos navigated by one global time-line. The right side bar contains buttons and text fields for creating user annotations.

marked and excluded from later analysis. The video annotation was performed by 4 raters and 177 data sets $(3.8 \%$ of the data) were annotated by all to calculate inter-rater reliability. A weighted Cohen's Kappa shows substantial agreement (linear: for users 0.65 , for actors 0.63 ; square: for users 0.8 , for actors 0.77 ). Note that users include both actors and spectators, while actors are only those who interact with the system at some point during the session. Each annotation was marked with a timestamp, allowing to find the position in the video for later reference and verification. Furthermore, we performed semi-structured interviews with 27 users during the last three days of deployment. The main objective was to find out if users were aware of interrupting their interaction and, in particular, what they did during this time. They were also asked whether they had seen and understood the hints that were displayed.

\section{RESULTS}

During our 10-day deployment 92,000 people were tracked as they passed the screens. A subset of 5,370 data sets was subsequently analyzed through video annotation, resulting in 704 annotated users. The following are our key findings.

\section{Quantitative findings}

\section{Number and duration of interruptions}

Actors (i.e., users who interacted with the screen at some point) interrupt an average of 1.13 times with an average interruption duration of 11.25 seconds. This confirms the finding from our meta-analysis that most users interrupt interaction at least once (RQ1). If we look at the interruption duration across techniques (shown in Figure 10), we notice that the longest average interruption duration was observed in the Quasimodal Pausing technique (not significant, $p=0.119$ ). The Amodal Activation technique shows a large variance with a maximum interruption duration that is way beyond that of the other techniques. However, this may be due to the difficulty of annotating interaction in the videos during this technique (since not much physical activity was required from the users). Furthermore, it is remarkable that Quasimodal Idling, which allows to transition between Active and Idle, appears to have shorter interruptions than Quasimodal Pausing, which allows transitions between Active and Paused. However, this effect is also not significant. 


\section{Duration of stay and interaction}

We found a significant influence of technique on duration of stay (one-way ANOVA: $F(3,700)=6.67, p<0.01$ ). A Tukey's pairwise comparison shows that the Amodal Activation technique had significantly longer durations of stay compared to all other techniques $(p<0.01)$ (see Figure 11). Similarly, there was a significant influence of technique on duration of interaction (one-way ANOVA: $F(3,491)=5.19, p<$ 0.01). A post-hoc pairwise Tukey's test reveals that the

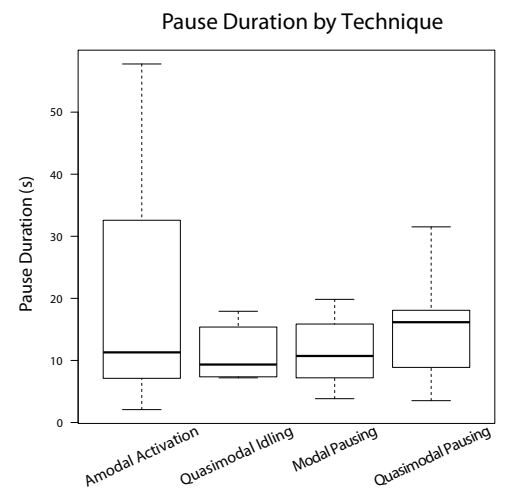

Figure 10. There was no significant effect of interruption duration by technique. However, we do see that interruptions appeared to be longest on average in Quasimodal Pausing.

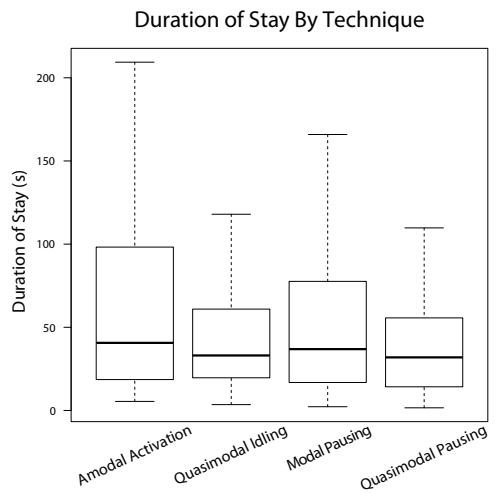

Figure 11. Duration of stay was significantly longer in the Amodal Activation technique compared to all other techniques.

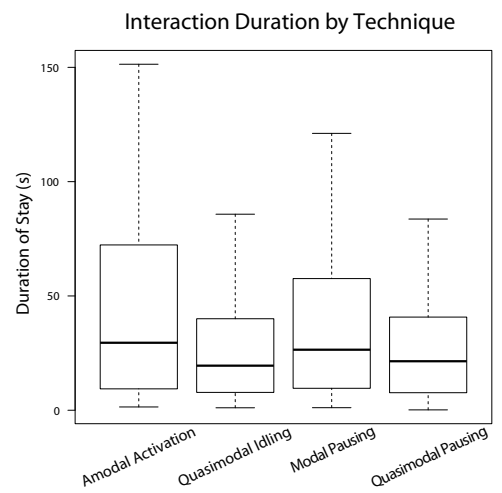

Figure 12. Interaction duration was significantly longer in the Amodal Activation technique compared to all other techniques.
Amodal Activation technique resulted in significantly longer interaction durations than all other techniques $(p<0.05)$ (see Figure 12). On average over all techniques actors spent 62 seconds interacting with the screen.

\section{Reasons for interrupting}

Users most often interrupted game-play for social interaction with others (161 reported cases). This was annotated during video analysis whenever social interaction was clearly visible (e.g., when users were seen talking, turning their heads towards each other, and/or gesturing towards each other or the screen). Sometimes interruptions appeared to be additionally encouraged by in-game events, e.g., when there were no enemies (166 cases), or events in the environment when tracking was lost, e.g., due to occlusion ( 77 cases). These events were clearly visible on the screen capture of the game, or the depth video from the Kinect. Other activities during interruptions include handling carried items, bags, and coats ( 27 cases) (see Figure 13), explaining the system and helping each other (11 cases), eating or drinking (5 cases), or dealing with the phone (4 cases). (RQ2)

\section{Users approach in groups}

We evaluated the typical group size of passers-by who stopped to interact, since this may impact turn-taking and social interaction. The average group size was 2.47 and users predominantly approached the screen in pairs (234 occurrences, 46\%), followed by single users (98 occurrences, $19 \%$ ), groups of 3 (92 occurrences, 18\%), and groups of 4 (45 occurrences, 9\%). Groups with 5 to 7 members, or more, were only rarely observed (5: 17 occurrences, 6: 11 occurrences, 7: 6 occurrences, 8: 7 occurrences). Larger groups became very difficult to annotate, due to the limited tracking area and crudeness of the Kinect's depth image, and were excluded from the analysis (uncertain group size: 194 occurrences)

\section{Qualitative findings}

Observations were made for several hours on a daily basis during our 10-day deployment. Additional observations stem from the analysis of our recorded video data.

\section{Modal Pausing technique rarely used to pause}

Through our video annotation we found that actors rarely bothered to explicitly pause the game. While $25 \%$ of all actors in the Modal Pausing technique triggered a pause, only very few seemed to do this with the intention of pausing, but most often merely appeared to explore the system's functionality, since they immediately continued interaction thereafter.

\section{Multitasking}

People often made an effort not to interrupt interaction while putting down a bag, taking off a jacket, eating or drinking. Instead, they attempted to interact simultaneously. We also observed that the T-pose was sometimes maintained, even though the actor was clearly distracted and not paying attention to the game.

\section{Courteous space management and turn-taking}

The members of a group usually spread out across both sets of screens. But when a new group arrived, the previous group 


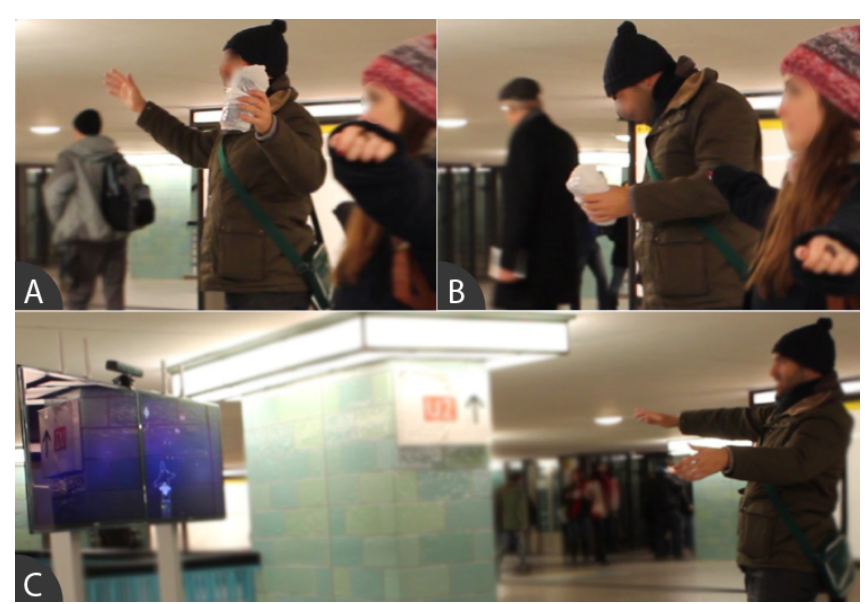

Figure 13. A user starts interacting with an item in his hand (A), then he interrupts interaction to place the item in his bag $(B)$ and resumes interaction $(\mathbf{C})$.

often withdrew to one set of screens, courteously leaving the other for the new arrivals. (This aligns with findings from an earlier installation called City Wall [25].) Furthermore, turntaking was frequently observed, with users ceasing interaction to watch their friends for a while and generously giving them space.

\section{Over-motivated and performative interaction}

The first user action was often to wave towards the displays. Also, people were found doing things that were not required or encouraged by the system: they performed, danced, played with their silhouettes and the silhouettes of others, or engaged in virtual fighting battles, kicking and punching each other's silhouettes. While performing the T-pose, users very often flapped their arms like a bird, or made rhythmic movements in time with the shooting interval. Furthermore they tilted their upper body, as if trying to aim their shots diagonally across the screen, which was not supported by the game.

\section{Interview results}

From our semi-structured interviews with 27 users we learned that almost all users noticed the hints that were displayed (23/27 users) and most of those found them helpful (21/22 users - 1 did not reply to the question). When asked how they knew how to play, about half of the users said that they followed the hints (13/27 users). The remaining either experimented (5/27 users), already knew the system (5/27 users), or learned through observation and explanation (4/27 users). About half of the users said they interrupted interaction at some point (13/27 users), with the most frequently stated reasons being trying to understand the game, doing the gesture wrong, fatigue, talking, or no enemies present. Only few of the users who reported interrupting interaction minded that their spaceship received damage during interruptions (4/13 users), with the remaining either being too distracted to notice (4/13 users), or simply not caring since "it's just a game" $(5 / 13)$. When asked about their reason for leaving, most reported that they had no more time (15/27 users), others found the game got boring ( $7 / 27$ users), and some said that their arms hurt (2/27 users).

\section{DISCUSSION}

Summarizing our main findings in regard to our three research questions:

- During our field study users did interrupt interaction for quite a long time. While spending an average of $62 \mathrm{sec}-$ onds in front of the screen, we found that users interrupt interaction 1.13 times on average, with an average interruption duration of 11.25 seconds. (RQ1)

- Users interrupted interaction most often to socialize with others, or also to explain the system to each other, handle coats, bags, and other carried items, eat or drink, deal with the phone, or when tracking was lost by the system. Examples can be seen in Figures 2 and 13. (RQ2)

- The play/pause gesture was rarely performed by users when interrupting interaction. (RQ3)

- In the Amodal Activation technique, which did not support pausing, the durations of stay and interaction were longest (Significant. See Figures 11 and 12). (RQ3)

Our research shows that people in groups are more likely to stop and engage with public displays than individuals passing by alone. Furthermore, social interaction within the group does not completely cease for the sake of interaction, but continues and can lead to divided attention of the user and intermissive interaction with the display. This may become a problem if the application on the public display requires continuous interaction and full attention, as for instance a fastpaced, dynamic game. Not handling this correctly may lead to frustrating experiences (like losing a game) and may discourage users from further interaction. Offering a pause mode therefore seems appropriate. But the interaction of groups must hereby be considered: if multiple users interact simultaneously and one user wishes to pause, how does this affect the other players? We have implemented the pause mode in such a way that each user can pause their own avatar and temporarily leave the game (Modal Pausing and Quasimodal Pausing). Hence, one person's pause means that the other players have more enemies to face themselves, but also more opportunity of shooting down spaceships and earning points. This could perhaps be better addressed in the future by dynamically adapting the number and strength of enemies to the number of players. A second challenge we faced was to find appropriate techniques for pausing interaction with gestures. The users had to learn these gestures through hints on the screen, by observation, or through instruction by other users. We chose the T-pose (ready pose) for the Quasimodal Pausing technique, since it is a metaphor for flying and the user's arms aligned with the spaceship's wings. In a different game, with other types of player representations and avatars, this trigger-pose should probably be adapted. The raise-hand gesture (play/pause gesture) was a more pragmatic choice, since it is easy to track and culturally a well known indication of participation. While our techniques were rarely used to pause the game, we observed users eagerly performing the gestures to explore the functionality of the system. This shows that they were aware of how to interact and were not hindered by the awkwardness of performing the gestures 
in public. Some people indicated that the T-pose was tiring and this may have led users to abandon interaction earlier. However, we think that a gesture that is both easy to detect and can be learned through observation, as well as being unusual enough to avoid inadvertent triggering, will necessarily be 'performative' and to some degree physically strenuous. We also think it is worth noting that the users' behavior of interrupting interaction was similar across all deployment locations (meta-analysis: shop window and university cafeteria, iterative design process: university campus, field study: subway station). This indicates that this phenomenon is not strongly influenced by the type of public setting.

\section{Users interrupt but don't pause}

We have found many different reasons for interruptions, the most common being social reasons (the user interrupts interaction to observe others, help others, engage in conversation, or to give someone space). Notably, similar interruptions were reported with the Flypad [9]. Furthermore we observed interruptions for personal reasons (the user interrupts interaction to eat, drink, put down a bag, take off a coat, take a picture, or answer a call), or due to the system (the user interrupts interaction because tracking is lost, or no enemies are present). Often multiple reasons coincide and are further influenced by non-observable factors like fatigue or puzzlement, which were occasionally mentioned in interviews. Our observations indicate that the reason for not pausing the game during these interruptions may be that users simply do not care enough about the contents of a public display. This aligns with earlier findings [24] and was confirmed in our interviews, where users mentioned occasionally that "it's just a game".

\section{Comparing our techniques}

We found the longest duration of both stay and interaction in the Amodal Activation technique (Figure 12 and 11), where no special action was required to play (the spaceships shoot automatically). So the absence of a manual pause mode, and thus the lack of an explicit action required from the user, appears to be beneficial. A possible explanation for shorter interaction times in all other techniques may be that the increased physical strain, required by the quasimode, caused people to abandon the screens more quickly. This could suggest that users are simply lazy. However, we frequently witnessed quite performative interaction, which seems to contradict this. Another reason might be that the Amodal Activation technique could more easily accommodate multiple people interacting simultaneously and is more robust to occlusion and tracking errors, since users do not need to perform the T-pose. Finally, it could be that, since this technique does not give users any chance to pause (users are constantly in Active state), the experience of flow in the game is greater.

Regarding interruption duration across techniques, it is interesting that there appears to be a difference between the two quasi modes (not significant, see Figure 10): Quasimodal Idling, which allows to transition between Active and Idle, shows interruptions that are on average 5 seconds shorter than Quasimodal Pausing, which allows transitions between Active and Paused. This might suggest that users actually take advantage of the opportunity to rest, if they can transition to Pause, while transitioning to Idle state (and thus still having a spaceship) keeps them more involved in the game.

In conclusion, we believe our results indicate that applications perform best if they require minimal effort to interact. Or in other words, the concept of a manual pause mode is too complex for a public display game and also to some degree hinders immediate usability. The application must take into account that the amount of attention and resources users are willing to dedicate to it vary, depending on social interactions and distractions from the environment, and it should be able to cope with interruptions, e.g., by tracking the user's attention, pausing automatically, and recovering gracefully.

\section{LIMITATIONS}

We believe our results could be useful for the development of casual games with body tracking in general (for instance on home consoles), and that they are not necessarily limited to public displays. It should be noted however that we have focused on full body interaction and that different forms of input (e.g., physical buttons, touch screen display) may prove better suited for offering a manual pause mode. We also wish to mention that the techniques we designed do not fully explore the design space and it remains for future research to evaluate if a different set of gestures provides comparable results. Furthermore, it would be interesting to evaluate pausing behavior in interaction with a different type of continuous application. Our game was intentionally kept simple to suit the nature of public display interaction (many first-time users, short interaction sessions) and for better generalizability, but introducing higher stakes for the players (e.g., with a less casual game, an ongoing competition, a serious task, etc.) may increase the users' need and appreciation of a pause mode.

\section{CONCLUSION}

The pause mode is a very central and useful component of most video games, where it is traditionally toggled by pressing a button or key. Newer input devices have led to other techniques for triggering the pause mode, such as the Guide Gesture for Kinect. Our studies show that users interrupt their playful interaction with public displays and do so most often for the sake of socializing. This allows the assumption that games on public displays would similarly profit from having a pause mode, affording controlled interruptions of the game. No previous research seems to have been done on the topic of interruptions on public displays, or more generally on pauses in games. To contribute to this underexplored field we performed a 10-day field study analyzing interruptions and evaluating four different pausing techniques in a simple public display game. However, the pause techniques we developed all had considerable downsides and we observed in our field study that, against our expectations, a manual pause mode is not well adopted by users when interrupting games on public displays. Therefore we conclude that the interaction with public displays should be made as simple as possible and interruptions should be implicitly supported by the application design, e.g., by ensuring that the application graciously recovers from interruptions. 


\section{ACKNOWLEDGMENTS}

Great thanks are due to the public transport company BVG and the Wall AG for making our field study in the subway station Alexanderplatz possible. We also wish to thank Ines Ben Said, Dieter Eberle, and Viktor Miruchna for their invaluable help with video annotation, as well as for their support during deployment along with Andreas Fender, Constantin Schmidt and Saiganesh Swaminathan.

\section{REFERENCES}

1. Florian Alt, Thomas Kubitza, Dominik Bial, Firas Zaidan, Markus Ortel, Björn Zurmaar, Tim Lewen, Alireza Sahami Shirazi, and Albrecht Schmidt. 2011. Digifieds: insights into deploying digital public notice areas in the wild. In MUM'11. ACM.

2. Elliot Aronson, Timothy D Wilson, Robin M Akert, and Beverly Fehr. 2012. Social psychology. Pearson Education Canada.

3. Alexander Biskupski, Andreas R Fender, Tiare M Feuchtner, Marcel Karsten, and Jonas D Willaredt. 2014. Drunken ed: a balance game for public large screen displays. In CHI'14 Extended Abstracts on Human Factors in Computing Systems. ACM, 289-292.

4. Philipp Brauner, André Calero Valdez, Ulrik Schroeder, and Martina Ziefle. 2013. Increase physical fitness and create health awareness through exergames and gamification. In Human Factors in Computing and Informatics. Springer, 349-362.

5. Harry Brignull and Yvonne Rogers. 2003. Enticing people to interact with large public displays in public spaces. In Proceedings of INTERACT, Vol. 3.

6. Xiang Cao, Michael Massimi, and Ravin Balakrishnan. 2008. Flashlight jigsaw: an exploratory study of an ad-hoc multi-player game on public displays. In CSCW'08. ACM.

7. Peter Dalsgaard and Kim Halskov. 2010. Designing urban media façades: cases and challenges. In CHI' 10. ACM.

8. Matthias Finke, Anthony Tang, Rock Leung, and Michael Blackstock. 2008. Lessons learned: game design for large public displays. In DIMEA'08. ACM.

9. Martin Flintham, Stuart Reeves, Patrick Brundell, Tony Glover, Steve Benford, Duncan Rowland, Boriana Koleva, Chris Greenhalgh, Matt Adams, Nick Tandavanitj, and others. 2011. Flypad: Designing trajectories in a large-scale permanent augmented reality installation. In ECSCW 2011: Proceedings of the 12th European Conference on Computer Supported Cooperative Work, 24-28 September 2011, Aarhus Denmark. Springer, 233-252.

10. Erving Goffman. 2008. Behavior in public places. Simon and Schuster.

11. Eva Hornecker, Paul Marshall, and Yvonne Rogers. 2007. From entry to access: how shareability comes about. In DPPI'O7. ACM.
12. Elaine M Huang, Anna Koster, and Jan Borchers. 2008. Overcoming assumptions and uncovering practices: When does the public really look at public displays? In Pervasive Computing. Springer.

13. Giulio Jacucci, Ann Morrison, Gabriela T Richard, Jari Kleimola, Peter Peltonen, Lorenza Parisi, and Toni Laitinen. 2010. Worlds of information: Designing for engagement at a public multi-touch display. In CHI' 10. ACM.

14. Hannu Kukka, Heidi Oja, Vassilis Kostakos, Jorge Goncalves, and Timo Ojala. 2013. What Makes You Click: Exploring Visual Signals to Entice Interaction on Public Displays. In CHI'13. ACM.

15. Annakaisa Kultima. 2009. Casual game design values. In MindTrek'09. ACM.

16. John J. Macionis and Ken Plummer. 2002. Sociology: A Global Introduction. Prentice Hall New York.

17. Jörg Müller, Dieter Eberle, and Konrad Tollmar. 2014. Communiplay: A Field Study of a Public Display Mediaspace. In CHI'14. ACM, New York, NY, USA.

18. Jörg Müller, Robert Walter, Gilles Bailly, Michael Nischt, and Florian Alt. 2012. Looking glass: A field study on noticing interactivity of a shop window. In CHI'12. ACM.

19. Jörg Müller, Dennis Wilmsmann, Juliane Exeler, Markus Buzeck, Albrecht Schmidt, Tim Jay, and Antonio Krüger. 2009. Display blindness: The effect of expectations on attention towards digital signage. In Pervasive Computing. Springer.

20. Ann Morrison and Antti Salovaara. 2008. Sustaining Engagement at a Public Urban Display. In $\mathrm{OzCHI}_{2} 08$.

21. Kenton O'Hara. 2003. Public and situated displays: Social and interactional aspects of shared display technologies. Vol. 2. Springer.

22. Kenton O'Hara, Maxine Glancy, and Simon Robertshaw. 2008. Understanding collective play in an urban screen game. In CSCW'08. ACM.

23. Timo Ojala, Vassilis Kostakos, Hannu Kukka, Tommi Heikkinen, Tomas Linden, Marko Jurmu, Simo Hosio, Fabio Kruger, and Daniele Zanni. 2012. Multipurpose interactive public displays in the wild: Three years later. Computer 45, 5 (2012).

24. Antti Oulasvirta, Sakari Tamminen, Virpi Roto, and Jaana Kuorelahti. 2005. Interaction in 4-second bursts: the fragmented nature of attentional resources in mobile HCI. In CHI'05. ACM.

25. Peter Peltonen, Esko Kurvinen, Antti Salovaara, Giulio Jacucci, Tommi Ilmonen, John Evans, Antti Oulasvirta, and Petri Saarikko. 2008. It's Mine, Don't Touch!: Interactions at a large multi-touch display in a city centre. In CHI'08. ACM. 
26. Jef Raskin. 2000. The humane interface: new directions for designing interactive systems. Addison-Wesley Professional.

27. Stuart Reeves, Steve Benford, Claire O'Malley, and Mike Fraser. 2005. Designing the spectator experience. In $C H I ' 15$. ACM.

28. M. Sherif and C.W. Sherif. 1948. Social psychology. Harper \& Row.

29. Oliver Storz, Adrian Friday, Nigel Davies, Joe Finney, Corina Sas, and Jennifer G Sheridan. 2006. Public ubiquitous computing systems: Lessons from the e-campus display deployments. Pervasive Computing, IEEE 5, 3 (2006).

30. Nick Taylor, Keith Cheverst, Dan Fitton, Nicholas JP Race, Mark Rouncefield, and Connor Graham. 2007. Probing communities: study of a village photo display. In OZCHI'07. ACM.
31. Nina Valkanova, Robert Walter, Andrew Vande Moere, and Jörg Müller. 2014. MyPosition: Sparking Civic Discourse by a Public Interactive Poll Visualization. In CSCW'14. ACM, New York, NY, USA.

32. Daniel Vogel and Ravin Balakrishnan. 2004. Interactive public ambient displays: transitioning from implicit to explicit, public to personal, interaction with multiple users. In UIST'04. ACM.

33. Robert Walter, Gilles Bailly, and Jörg Müller. 2013. StrikeAPose: Revealing Mid-Air Gestures on Public Displays. In CHI'13. ACM.

34. Robert Walter, Gilles Bailly, Nina Valkanova, and Jörg Müller. 2014. Cuenesics: Using Mid-Air Gestures to Select Items on Interactive Public Displays. In MobileHCI'14. ACM. 